\section{OC57 VON WILLEBRAND DISEASE RECLASSIFICATION IN A NATIONAL PAEDIATRIC COMPREHENSIVE CARE CENTRE}

Irene E Regan*, Bridin Brady, Mary Kavanagh, Imelda Kelly, Beatrice Nolan. Coagulation/ Haematology, Our Lady's Children's Hospital Crumlin, Dublin, Ireland

\subsection{6/archdischild-2019-epa.54}

In 2008, the National Institutes of Health published guidelines (www.nhlbi.nih.gov/health-pro/guidelines/current/von-willebrandguidelines/full-report/4-management-of-vwd.htm) in which the suggested level for the designation of type 1 VWD was a VWF:Ag or VWF:RCo level of $<30 \mathrm{IU} / \mathrm{dL}$ and 'low VWF levels' refer to individuals with VWF:Ag levels between 30 and $50 \mathrm{IU} / \mathrm{dL}$. The aim was to reclassify the VWD patient cohort at the National Paediatric Comprehensive Care Centre Our Lady's Children's Hospital Crumlin, Dublin.

Methods 315 Case records of children $<18$ years with VWD or possible VWD over a 10 year period were retrospectively extracted from the Irish National Bleeding Disorder database. These records were 'interpreted' according to NHLBI/NIH diagnostic criteria. The algorithm applied was; VWF level$\mathrm{s}<30 \mathrm{IU} / \mathrm{dl}$ on 2 separate occasions-VWD; VWF levels 30$50 \mathrm{IU} / \mathrm{dl}$ on 2 separate occasions-Low VWF; VWF not less than 50IU/dl on 2 occasions and multiple testing-not VWD. Blood group was also recorded. Where incomplete laboratory data, patients were recalled to a review clinic for further testing.

Results 315 children on the Irish National Bleeding disorder database had been historically diagnosed with VWD or possible VWD.

Following the review there was a $81 \%$ reduction in the number of patients diagnosed with Type 1 VWD (187 patients in the original cohort were classified as Type 1 which reduced to 36 post -reclassification).

Predictably, no significant change in numbers diagnosed with Type 2 (26) and Type 3 (2 patients) VWD,

185 (59\% of total population) are now classified as Low VWF with a preponderance of Group $\mathrm{O}$ patients.

37 (15\% of total population) were deemed to have no form of VWD and reclassified as normal.

19 (6\% of total population) have not returned for full reclassification and still remain as unspecified or possible.

Discussion/Conclusion Our data suggest over-diagnosis of VWD in this population using previous guidelines. Diagnosis, especially for individuals with mildly decreased VWF (30$50 \mathrm{IU} / \mathrm{dl}$ ) requires correlation of clinical assessment and laboratory results. Reclassification has resulted in reallocation of resources to priority patients.

This recommendation does not preclude the diagnosis of VWD in individuals with VWF:RCo of $30-50 \mathrm{IU} / \mathrm{dl}$ if there is supporting clinical and/or family evidence for VWD or the use of agents to increase VWF levels where VWF:RCo is 30$50 \mathrm{IU} / \mathrm{dl}$ and may be at risk for bleeding.

\section{OC58 MORBIDITY AND MORTALITY OF MEDICAL AND SURGICAL NECROTISING ENTEROCOLITIS}

1Janet Lang*, 1,2 Leah Hickey, 1,2 Sebastian King, ${ }^{3}$ Anna Curley. ${ }^{1}$ Royal Children's Hospital, Melbourne, Australia; ${ }^{2}$ The University of Melbourne, Melbourne, Australia; ${ }^{3}$ The National Maternity Hospital, Dublin, Ireland

10.1136/archdischild-2019-epa.55
Introduction Necrotising enterocolitis (NEC) is the most serious and frequent gastrointestinal emergency in the neonatal intensive care unit, (NICU) and is a major cause of death in premature infants. It is also associated with considerable morbidity, including increased respiratory support and level of care, need for intravenous antibiotics and total parenteral nutrition (TPN), and has long-term effects on growth and neurodevelopment.

Aim This study aims to evaluate the difference in mortality and short-term morbidity between infants with medically and surgically treated NEC in a tertiary-level surgical neonatal unit in Cambridge, England.

Methods This retrospective analysis of prospectively collected data evaluated infants with a diagnosis of NEC between 1st January 2009 and 31st December 2011. Diagnosis was made using modified Bells criteria and infants were defined into two groups by the treatment received. Medical NEC was treated with standard therapy of 7 days of broad spectrum intravenous antibiotics and withholding of enteral feeding, while surgical NEC was defined as the requirement for operative intervention; either laparotomy or placement of a peritoneal drain.

Results During the 3-year study period, 152 infants were diagnosed with NEC and met inclusion criteria. Of these, 82 required medical management only and 70 required surgical intervention in addition to the standard medical treatment. There was a significant difference in mortality between the two groups with survival in the medically managed group of $96 \%$ vs. $61 \%$ in the operative group $(\mathrm{p} \leq 0.0001)$. The surgical group had a statistically significant higher rate of NEC recurrence $(14 \%$ versus $28.5 \%, p=0.03)$. The surgical group had more days of ventilation ( 7 vs $13.5, \mathrm{p}=0.001$ ), more days on TPN (26 vs $46 \mathrm{p}<0.0001)$ and were more likely to receive surgical central line insertion under general anaesthetic $(11 \%$ vs $26 \%$, $\mathrm{p}=0.02$ ). There were no significant differences in gestation or birth weight, type of feeding or early somatic growth.

Conclusion This is the largest single centre study comparing these two treatment groups and provides accurate contemporaneous data with which to counsel families. Operative NEC is associated with greater mortality and a higher rate of recurrence when compared with medical NEC. There is also significant morbidity associated with surgical NEC, including longer ventilation and long-term use of total parenteral nutrition, which has associated complications and sequelae.

\section{OC59 MULTIMODAL MONITORING AS PREDICTOR OF BRAIN INJURY IN THE PRETERM INFANT}

${ }^{1}$ David O'Sullivan*, ${ }^{2}$ John O'Toole, ${ }^{2}$ Daragh Finn, ${ }^{2}$ Aisling Garvey, ${ }^{2}$ Andreea Pavel, ${ }^{2}$ Vicki Livingstone, ${ }^{2}$ Geraldine Boylan, ${ }^{1,2}$ Eugene Dempsey. ${ }^{1}$ Paediatrics and Child Health, University College Cork, Cork, Ireland; ${ }^{2}$ INFANT Centre, Cork, Ireland

\subsection{6/archdischild-2019-epa.56}

Background Cerebral auto-regulation(CAR) in the preterm infant is a complex, multi-factorial process which is still poorly characterised in very preterm infants. It plays a substantial role in the aetiology of intraventricular haemorrhage (IVH). Studies typically examine the relationship between cerebral oxygenation measured using near-infrared spectroscopy (NIRS) and mean arterial blood pressure(MABP) as a method to study CAR impairment.

Objective To explore a more comprehensive, multi-modal method of analysis for CAR that incorporates other potentially important factors such as continuous electroencephalogram (cEEG) and cardiac output(CO) measurements. 
Design/Methods A secondary analysis of prospectively gathered data from a randomised trial of different cord management strategies. 38 preterm infants were monitored between 6 and 18 hours after birth. Echocardiogram measurements of right and left ventricular output(RVO, LVO) and superior vena cava flow(SVC) were performed. cEEG and changes in cerebral blood oxygenation were determined by NIRS at 6 and 12 hour timepoints. Development and grade of IVH was assessed by cranial ultrasound (CRUS) at 24hours. Quantitative features were determined for cEEG and NIRS values. Spearman rank correlations were calculated between RVO, LVO, SVO and the NIRS and EEG quantitative features in infants with and without IVH.

Results Of the 38 infants analysed(median GA 28.0 weeks [23.6-31.6], median BW 950 g [530-2040 g]) 13 preterm infants developed IVH within 24 hours. Following analysis, a notable difference in relations between LVO, RVO and EEG quantitative features was found between those infants with and without IVH. Correlations of EEG features such as IBI Length $\max (r=0.71$, pvalue 0.01$)$, IBI burst $\% \quad(r=-0.61$, pvalue 0.05$)$ and rEEG asymmetry $(r=0.74$, $p$ value 0.01$)$ with LVO showed significant relationships at both $6 \mathrm{hrs}$ and again IBI Length max $(r=0.71$, pvalue 0.01$)$, IBI burst $\% \quad(r=-0.66$, pvalue 0.05$)$ and rEEG asymmetry $(r=0.65$, p value 0.05$)$ at $12 \mathrm{hr}$ timpeoints in the IVH subgroup. No significant correlations were found between NIRS and CO measures in both groups.

Conclusion(s) Correlation of early continuous EEG quantitative data with LVO measures demonstrated a significant difference in features between preterm infants with IVH and those without. These results may indicate that incorporation of $\mathrm{CO}$ measurements and cEEG into a multi-modal method of neonatal monitoring may permit early identification of preterm infants at increased risk of IVH.

\section{OC60 EVALUATION OF PERINATAL DEVELOPMENT OF OFFSPRING BORN TO MOTHERS WITH GESTATIONAL DIABETES}

Irirna Nikitina*, Alisa Masel, Ekaterina Kaprior, Daria Yuablunovskaya, Igor Kelmanson. Almazov National Medical Research Centre, Saint Petersburg, Russian Federation

\subsection{6/archdischild-2019-epa.57}

Background According to The Hyperglycemia and Adverse Pregnancy Outcome Study (HAPO Study) 2008, hyperglycemia caused by maternal gestational diabetes mellitus (GDM) is an important contributor to adverse fetal programming and maternal complications.

Aim To assess the associations between the GDM and perinatal outcomes.

Design The study comprised 228 women who were divided into two groups: Group 1 (Gr1) included 157 women with GDM, group 2 (Gr2) - 71 women without GDM. The diagnosis of GDM has been confirmed according to the HAPO criteria. Retrospective analysis of pregnancy, delivery and early neonatal period were performed. Newborn anthropometric characteristics were assessed and interpreted according to INTERGROWTH-21 ${ }^{\text {st }}$ recommendations. Statistical significance was estimated using Mann-Whitney $U$ and chisquared tests.

Results The rate of vaginal deliveries in both groups was similar (Gr1 81.7\%; Gr2 73.5\%, p = 0.19). The presence of
GDM was associated with an increased risk of obstetric injuries (OR 4.1, 95\% CI 1.9-8.5; p = 0.00004) and hypoglyce$\mathrm{mia}$ in the newborn (OR 5.4, 95\% CI 2.2-13.1; $\mathrm{p}=$ 0.00002). No significant differences were found in birth weight (Gr1: Me $3500 \pm 400 \mathrm{~g}$ vs Gr2: Me $3450 \pm 500 \mathrm{~g}$, $\mathrm{p}=0.7$ ), birth length (Gr1: Me $52 \pm 0.2 \mathrm{~cm}$ vs Gr2: Me $52 \pm 0.2 \mathrm{~cm}, \mathrm{p}=0,3$ ), weight/length ratio (Gr1: Me $6.7 \pm$ $0.5 \mathrm{~kg} / \mathrm{cm}$ vs Gr2: Me $6.6 \pm 0.7 \mathrm{~kg} / \mathrm{cm}, \mathrm{p}=0.9)$ and head circumference (Gr1: Me $35.2 \pm 2.3 \mathrm{~cm}$ vs Gr2: Me $35.0 \pm$ $1.3 \mathrm{~cm}, \mathrm{p}=0.05)$. The risk of being born with 'upper average' weight was associated with presence of maternal GDM ( $>1$ SDS $<2$ SDS) (OR 2.47; 95\% CI 1.2-5.1; p $=0.01)$. However, no association was found between GDM and neonatal weight exceeding 2 SDS, known as 'macrosomia' (OR 1.1; 95\% CI $0.3-3.7 ; p=0.83)$. Maternal GDM was also associated with increased risk of neonatal head circumference in the 'upper average' range (> 1 SDS) (OR 2.4; 95\% CI 1.4-5.9; $\mathrm{p}=0.005)$.

Conclusion Maternal GDM was associated with increased risk of obstetric injuries, which can be explained by higher rates of increased newborn weight and head circumference. However, there was no increased number of children with macrosomia born to GDM mothers, which indicates a limited impact of GDM on fetal development compared to diabetes type 1 or 2 . Neonatal hypoglycemia was associated with maternal GDM as well.

\section{OC61 A SYSTEMATIC REVIEW OF SERUM BIOMARKERS IN NEONATAL ENCEPHALOPATHY TO PREDICT SHORT TERM OUTCOME}

1,2,3Mary O'Dea*, ${ }^{1}$ Tim Hurley, ${ }^{4}$ David Mockler, ${ }^{5}$ Kasper Kyng, ${ }^{6}$ Phillips Bob, 2,7,8,9Eleanor Molloy. ${ }^{1} 1$. Department of Paediatric and Child Health Trinity College Dublin, Dublin, Ireland; ${ }^{2}$ Coombe Women and Infant's University Hospital, Dublin, Ireland; ${ }^{3}$ National Children's Research Centre, Crumlin, Dublin, Ireland; ${ }^{4} 2$. Subject Librarian, School of Medicine, Trinity College Dublin, Dublin, Ireland; ${ }^{5}$. University of Aarhus, Aarhus, Denmark; ${ }^{6} 4$. University of York, York, UK; ${ }^{7}$ Department of Paediatric and Child Health Trinity College Dublin, Dublin, Ireland; ${ }^{8}$ Our Lady's Childrens Hospital, Crumlin, Dublin, Ireland; ${ }^{9}$ National Children's Hospital, Tallaght, Dublin, Ireland

\subsection{6/archdischild-2019-epa.58}

Background Neonatal Encephalopathy (NE) describes central nervous system dysfunction from all causes and has a multifactorial aetiology. NE is difficult to diagnose, to treat and to predict outcome. Early prognostic information is important to initiate early intervention of therapies, to counsel parents and to redirect care. There is no gold standard early biomarker to predict outcome at present.

Methods The review protocol was prospectively registered with Prospero. A comprehensive search with relevant search terms of online databases Em Base, PubMed, Cochrane and Web of Science for Biomarkers in Neonatal Encephalopathy to Predict outcome was performed. The short term outcomes examined include severity of Neonatal Encephalopathy, MRI brain and survival in the neonatal period.

Two independent reviewers used Covidence software to select the studies. Quality assessment was performed using Cochrane Collaboration Tool for Risk of Bias Assessment and quantitative analysis was completed using Revman software (5.3).

Results and Discussion 1613 papers were identified after duplicates were excluded, and narrowed to 314 papers following abstract review for full text examination. Following full text 